Mathematical Modelling and Analysis

Volume 17 Number 1, February 2012, 128-139

http://dx.doi.org/10.3846/13926292.2012.647100

(c) Vilnius Gediminas Technical University, 2012
Publisher: Taylor\&Francis and VGTU

http://www.tandfonline.com/TMMA

Print ISSN: 1392-6292

Online ISSN: 1648-3510

\title{
Strictly Convergent Algorithm for an Elliptic Equation with Nonlocal and Nonlinear Boundary Conditions*
}

\section{Karlis Birgelis and Uldis Raitums}

\author{
Faculty of Physics and Mathematics, University of Latvia \\ Zellu st. 8, LV-1002 Riga, Latvia \\ E-mail(corresp.): k.birgelis@gmail.com \\ E-mail: uldis.raitums@mii.lu.lv
}

Received August 30, 2011; revised December 1, 2011; published online February 1, 2012

\begin{abstract}
The paper describes a formally strictly convergent algorithm for solving a class of elliptic problems with nonlinear and nonlocal boundary conditions, which arise in modeling of the steady-state conductive-radiative heat transfer processes. The proposed algorithm has two levels of iterations, where inner iterations by means of the damped Newton method solve an appropriate elliptic problem with nonlinear, but local boundary conditions, and outer iterations deal with nonlocal terms in boundary conditions.
\end{abstract}

Keywords: conductive-radiative heat transfer, elliptic equation, Newton method.

AMS Subject Classification: $35 \mathrm{~J} 65$.

\section{Introduction}

Various technological processes involve, as an important part, conductiveradiative heat transfer due the high temperatures characterizing the process. We mention only few of them: glass fabric production [3, 4]; glass melting and fining [8]; crystal growth in the production of semiconductors [10]. The corresponding mathematical model for conductive-radiative steady-state heat transfer was suggested and investigated by M. Laitinen and T. Tiihonen in [9], where the solvability of the state equation and boundedness of solutions for practically interesting data was given (see also $[3,10]$ ).

The standard mathematical model for the steady-state conductive-radiative heat transfer in a system with simple geometry (see Fig. 1) according to [9] is

* This work was supported by the Latvian Council of Sciences under grant 09.1572 and by the ESF project No. 2009/0223/IDP/1.1.1.2.0/APIA/VIAA/008. 


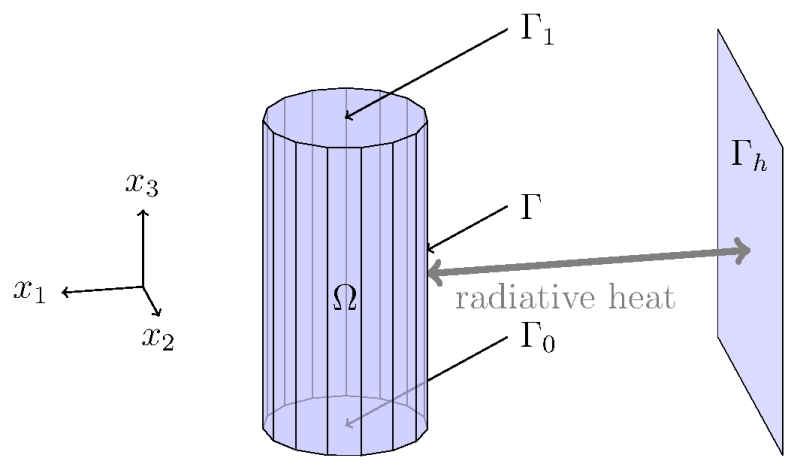

Figure 1. Geometry of the problem.

given by the variational equality

$$
\begin{gathered}
\int_{\Omega}\left[a\langle\nabla u, \nabla \eta\rangle+b u_{x_{3}} \eta\right] d x+\sigma \int_{\Gamma}\left[(I-H)\left(|u|^{3} u\right)\right] \eta d S \\
=\langle\langle\tilde{f}, \eta\rangle\rangle \quad \forall \eta \in W_{2}^{1}(\Omega) \cap L_{5}(\Gamma) \cap\left\{\eta=0 \text { on } \Gamma_{0}\right\},
\end{gathered}
$$

where nonlocal operator $H \in \mathcal{L}\left(L_{5 / 4}(\Gamma) \rightarrow L_{5 / 4}(\Gamma)\right)$ has the following representation for the case of a diffuse gray surface $\Gamma \cup \Gamma_{h}$ :

$$
[H(v)](x)=\left[\left((1-\epsilon) I+\epsilon K(I-(1-\epsilon) K)^{-1} \epsilon\right)\left(\begin{array}{l}
v \\
0
\end{array}\right)\right](x) \quad v \in L_{5 / 4}(\Gamma), x \in \Gamma .
$$

Here $\epsilon(x) \in(0,1]$ is emissivity distribution on $\Gamma \cup \Gamma_{h}, I$ is the identity operator and $K \in \mathcal{L}\left(L_{5 / 4}\left(\Gamma \cup \Gamma_{h}\right) \rightarrow L_{5 / 4}\left(\Gamma \cup \Gamma_{h}\right)\right)$ is an integral operator defined as

$$
\begin{gathered}
{\left[K\left(\begin{array}{l}
v_{1} \\
v_{2}
\end{array}\right)\right]\left(\begin{array}{l}
x \\
y
\end{array}\right)=\left(\begin{array}{l}
\int_{\Gamma} k(x, t) v_{1}(t) d S+\int_{\Gamma_{h}} k(x, z) v_{2}(z) d S \\
\int_{\Gamma} k(y, t) v_{1}(t) d S+\int_{\Gamma_{h}} k(y, z) v_{2}(z) d S
\end{array}\right)} \\
v_{1} \in L_{5 / 4}(\Gamma), v_{2} \in L_{5 / 4}\left(\Gamma_{h}\right), x, t \in \Gamma, y, z \in \Gamma_{h},
\end{gathered}
$$

where for arbitrary points $r, s \in \Gamma \cup \Gamma_{h}$

$$
k(r, s)=\frac{\cos (n(r),(s-r)) \cos (n(s),(r-s))}{\pi|r-s|^{2}} \theta(r, s) .
$$

Here $\theta(r, s) \in\{0 ; 1\}$ denotes visibility factor, $n(r), n(s)$ are normal unit vectors to $\Gamma \cup \Gamma_{h}$.

Despite the good solvability properties of (1.1), till now in literature there are not, as far as we know, described any rigorously convergent algorithm for this equation. The aim of this paper is to give a theoretically strictly convergent iterative procedure for solving the problem (1.1).

The key assumptions in our approach are that the operator $H$, caused by the radiative process, is a contraction in $L_{1}(\Gamma)$ due to "openness" of the surface $\Gamma$ (see, for instance, [9]) and that the "good geometry" of the system ensures 
necessary solvability of the Neumann problem for the Poisson equation in $\Omega$ with right hand sides in spaces $L_{p}\left(\Omega ; \mathbb{R}^{3}\right)$ with $p \in[4 / 3,4]$.

The paper is organized as follows. In Section 2 we give the precise statement of our problem and involved assumptions. In Section 3 we reduce our initial problem to a more convenient one with linear growth at infinity of the nonlinearity in the Stefan-Boltzmann law. In Section 4 we investigate the linearized equation, show the uniform invertibility of the corresponding operator and in Section 5 we show how this property ensures the convergence of a certain Newton type iterative process. In Section 6 we present a strictly convergent two level iterative procedure, where the basic procedure consists of solving a standard boundary value problem for a linear elliptic equation. Finally, in Section 7 we present some numerical results to illustrate performance of the proposed iterative procedure.

\section{Statement of the Problem}

Let $\Sigma_{0} \subset \mathbb{R}^{2}$ be bounded Lipschitz domain homeomorphic to the unit circle and let $\Omega$ be the cylinder $\Omega:=\Sigma_{0} \times(0, L) \subset \mathbb{R}^{3}$ with points $\left(x_{1}, x_{2}, x_{3}\right)$. The bottom of $\Omega$ we shall denote by $\Gamma_{0}, \Gamma_{0}:=\bar{\Omega} \cap\left\{x_{3}=0\right\}$, the upper part of $\Omega$ we shall denote by $\Gamma_{1}, \Gamma_{1}:=\bar{\Omega} \cap\left\{x_{3}=L\right\}$, and the lateral surface of $\Omega$ we shall denote by $\Gamma$ (see Fig. 1).

In what follows we suppose that the following hypothesis holds.

$\mathbf{H}_{\mathbf{0}}$. There exists a constant $c_{0}$ such that for all $4 / 3 \leq p \leq 4$ and for every $\bar{f} \in L_{p}\left(\Omega ; \mathbb{R}^{3}\right)$ the variational equality

$$
\int_{\Omega}\langle\nabla v, \nabla \eta\rangle d x=\int_{\Omega}\langle\bar{f}, \nabla \eta\rangle d x \quad \forall \eta \in W_{p^{\prime}}^{1}(\Omega), \text { with } \eta(\cdot)=0 \text { on } \Gamma_{0}
$$

has a unique solution $u \in W_{p}^{1}(\Omega)$ with $u(\cdot)=0$ on $\Gamma_{0}$ that satisfies

$$
\left(\int_{\Omega}|\nabla u|^{p} d x\right)^{\frac{1}{p}} \leq c_{0}\left(\int_{\Omega}|\bar{f}|^{p} d x\right)^{\frac{1}{p}} .
$$

Here and throughout this paper by $\langle\cdot, \cdot\rangle$ we denote the scalar product in $\mathbb{R}^{3}$ and by $\langle\langle\cdot, \cdot\rangle\rangle$ - the pairing between a Banach space $X$ and its dual space $X^{*}$.

The hypothesis $H_{0}$ holds, for instance, if $\Sigma_{0}$ has a $C^{1}$ boundary or $\Sigma_{0}$ is a rectangle. These properties are straightforward consequences from Theorem 4 [6, p. 16], which gives the necessary property for bounded domains with $C^{1}$ boundary in spaces $\mathbb{R}^{n}$. Our case with smooth boundary of $\Sigma_{0}$ is easily reducible to that by using simple mirror reflections with respect to the planes $\left\{x_{3}=0\right\},\left\{x_{3}=L\right\},\left\{x_{3}=-L\right\}$ and transform to the case where the vectorfunction $\bar{f}$ is equal to zero in a neighborhood of the top and the bottom of our cylinder $\Omega$. The case with rectangular $\Sigma_{0}$ is treated analogously.

Let us denote, for $1<p<\infty$, a space $V_{p}$ :

$$
V_{p}:=\left\{v \in W_{p}^{1}(\Omega) \mid v=0 \text { on } \Gamma_{0} \text { in the sense of embedding theorems }\right\}
$$

with the norm

$$
\|v\|_{p}:=\left(\int_{\Omega}|\nabla v|^{p} d x\right)^{\frac{1}{p}}
$$


Let the operator $H: L_{1}(\Gamma) \rightarrow L_{1}(\Gamma)$ satisfies the following hypotheses.

$\mathbf{H}_{\mathbf{1}}$. The operator $H$ belongs to $\mathcal{L}\left(L_{p}(\Gamma) \rightarrow L_{p}(\Gamma)\right)$ for all $1 \leq p \leq \infty$;

$\mathbf{H}_{\mathbf{2}}$. $\|H\|_{\mathcal{L}\left(L_{1}(\Gamma) \rightarrow L_{1}(\Gamma)\right)}=q<1$.

Consider the problem (with respect to $u \in V_{2} \cap L_{5}(\Gamma)$ )

$$
\begin{aligned}
\int_{\Omega} & {\left[\left\langle a \nabla\left(u+u_{*}\right), \nabla \eta\right\rangle+b\left(u+u_{*}\right)_{x_{3}} \eta\right] d x } \\
& +\sigma \int_{\Gamma}\left[(I-H)\left(\left|u+u_{*}\right|^{3}\left(u+u_{*}\right)\right)\right] \eta d S \\
= & \int_{\Omega} f_{0} \eta d x+\int_{\Gamma} g \eta d S \quad \forall \eta \in V_{2} \cap L_{5}(\Gamma),
\end{aligned}
$$

where the parameters $a, b, \sigma$ and functions $u_{*}, f_{0}, g$ satisfy the hypothesis

$\mathbf{H}_{3} . a>0, b \geq 0, \sigma>0, f_{0} \in L_{2}(\Omega), g \in L_{\infty}(\Gamma), u_{*} \in W_{4}^{1}(\Omega)$.

Analogous to (2.1) problems arise in conductive-radiative heat transfer, see, for instance, M. Laitinen and T. Tiihonen [9], K. Birgelis [3], C. Mayer and others [10] and references therein.

\section{Modified Problem}

In $[3,9]$ it was shown that if functions $f_{0}, g, u_{*}$ belong to a given set $M_{0}$, elements of which are of practical interest, then the solutions $u_{0}$ of (2.1) are bounded and there exists a constant $u_{\max }$ such that

$$
0 \leq u_{0}(x)+u_{*}(x) \leq u_{\max } \quad \forall x \in \bar{\Omega} .
$$

More than that, by using special truncated trial functions

$$
\eta_{\tau}(x):= \begin{cases}1, & \text { if } \eta(x) \geq \tau, \\ \eta(x) / \tau, & \text { if }-\tau<\eta(x)<\tau, \\ -1, & \text { if } \eta(x) \leq-\tau,\end{cases}
$$

in [3] it was shown that the "linearized" homogeneous problem

$$
\int_{\Omega}\left[a\langle\nabla v, \nabla \eta\rangle+b v_{x_{3}} \eta\right] d x+\sigma \int_{\Gamma}[(I-H)(\alpha v)] \eta d S=0 \quad \forall \eta \in V_{2}
$$

with an arbitrary nonnegative $\alpha \in L_{\infty}(\Gamma)$ has only a trivial solution in $V_{2}$.

Therefore, for cases of practical interest we can pass to the modified problem with respect to $u \in V_{2}$

$$
\begin{aligned}
& \int_{\Omega}\left[a\left\langle\nabla\left(u+u_{*}\right), \nabla \eta\right\rangle+b\left(u+u_{*}\right)_{x_{3}} \eta\right] d x+\sigma \int_{\Gamma}\left[(I-H)\left(\psi\left(u+u_{*}\right)\right)\right] \eta d S \\
& =\langle\langle f, \eta\rangle\rangle \quad \forall \eta \in V_{2},
\end{aligned}
$$

where the element $f \in\left(V_{2}\right)^{*}$ is given and the function $\psi$ satisfies the hypothesis 
$\mathbf{H}_{\mathbf{4}} \cdot \psi \in C^{1,1}(\mathbb{R})$ and there are constants $u_{\max }, \mu_{1}, \mu_{2}$ such that

$$
\begin{aligned}
& 0 \leq \psi^{\prime}(t) \leq \mu_{1} \quad \forall t \in \mathbb{R} \\
& \psi(0)=0, \quad \psi^{\prime}(t)=\mu_{1}, \quad \text { if }|t| \geq u_{\max } \\
& \left|\psi^{\prime}\left(t_{1}\right)-\psi^{\prime}\left(t_{2}\right)\right| \leq \mu_{2}\left|t_{1}-t_{2}\right| \quad \forall t_{1}, t_{2} \in \mathbb{R} .
\end{aligned}
$$

By construction, for cases of practical interest the modified problem (3.3) has the same solution $u_{0}$ as the original problem (2.1). Later we shall show that under hypotheses $H_{0}-H_{4}$ the problem (3.3) has the unique solution. Hence, in what follows we shall consider the problem (3.3) under hypotheses $H_{0}-H_{4}$.

\section{Linearized Equation}

Let us denote, for fixed constants $\mu$ and $\theta=0$ or 1 , by $\mathbf{A}(\mu)$ the set of linear operators

$$
\mathcal{A}_{\alpha}: V_{p} \rightarrow\left(V_{p^{\prime}}\right)^{*}, \quad p^{\prime}:=\frac{p}{p-1}
$$

defined as

$$
\begin{aligned}
\left\langle\left\langle\mathcal{A}_{\alpha}(v), \eta\right\rangle\right\rangle:= & \int_{\Omega}\left[a\langle\nabla v, \nabla \eta\rangle+b v_{x_{3}} \eta\right] d x \\
& +\sigma \int_{\Gamma} \alpha v \eta d S-\sigma \theta \int_{\Gamma} H(\alpha v) \eta d S \quad \forall \eta \in V_{p^{\prime}}
\end{aligned}
$$

with some $\alpha \in L_{\infty}(\Gamma), 0 \leq \alpha \leq \mu$. Our most interest is for $p=2$ and $p=4 / 3$.

From hypothesis $H_{0}$ it follows that $\left(V_{p}\right)^{*}$ is isometrically isomorphic to $V_{p^{\prime}}$ for $4 / 3 \leq p \leq 4$, see, for instance, Adams [1, Th. 3.10, p. 50]. Since the spaces $V_{p}, 1<p<\infty$ are reflexive (as closed linear subspaces of reflexive spaces), see, Adams [1, 1, Th. 35, p.47], then the conjugate operator $\left(\mathcal{A}_{\alpha}\right)^{*}: V_{p^{\prime}} \rightarrow\left(V_{p}\right)^{*}$ has the representation

$$
\begin{aligned}
\left\langle\left\langle\mathcal{A}_{\alpha}^{*}(\eta), v\right\rangle\right\rangle:= & \int_{\Omega}\left[a\langle\nabla v, \nabla \eta\rangle+b v_{x_{3}} \eta\right] d x \\
& +\sigma \int_{\Gamma} \alpha v \eta d S-\sigma \theta \int_{\Gamma} H(\eta) \alpha v d S \quad \forall v \in V_{p} .
\end{aligned}
$$

Lemma 1. All operators $\mathcal{A}_{\alpha} \in \mathbf{A}(\mu)$ are invertible.

Proof. To begin with, we show that all operators $\mathcal{A}_{0}$ are invertible (we remind that we consider only exponents $4 / 3 \leq p \leq 4$ and the operators $\mathcal{A}_{0}$ for $\alpha=0$ are defined by $(4.1)$ with $\theta=0)$. Indeed, we can represent the operator $\mathcal{A}_{0}^{*}$ as $\mathcal{A}_{0}^{*}=\mathcal{A}_{1}^{*}+\mathcal{A}_{2}^{*}$, where

$$
\left\langle\left\langle\mathcal{A}_{2}^{*}(\eta), v\right\rangle\right\rangle:=\int_{\Omega} b \eta v_{x_{3}} d x \quad \forall v \in V_{p} .
$$

Due the embedding theorems, the operator $\mathcal{A}_{2}^{*}$ is completely continuous and due our hypothesis $H_{0}$ the operator $\mathcal{A}_{1}^{*}$ is invertible. Hence, we can apply the Fredholm alternative to $\mathcal{A}_{0}^{*}$. 
If $p \leq 2$, we can in the equation

$$
\int_{\Omega}\left[a\langle\nabla \eta, \nabla v\rangle+b \eta v_{x_{3}}\right] d x=0 \quad \forall v \in V_{p}
$$

insert as $v$ the function $\eta$ what will give that $\eta=0$. That is sufficient for the invertibility of $\mathcal{A}_{0}^{*}$.

In turn, if $p \geq 2$, analogous reasoning we apply to the operator $\mathcal{A}_{0}$. This way, the operators $\mathcal{A}_{0}$ and $\mathcal{A}_{0}^{*}$ are invertible.

Further, due the embedding theorems and $H_{1}$, the operators $\mathcal{A}_{\alpha}-\mathcal{A}_{0}$ and $\mathcal{A}_{\alpha}^{*}-\mathcal{A}_{0}^{*}$ are completely continuous. Thus, again, it is sufficient to show that the equation $\mathcal{A}_{\alpha} v=0$ (or $\mathcal{A}_{\alpha}^{*} \eta=0$ ) has only the trivial solution.

If $p \geq 2$, we can in the integral equality

$$
\left\langle\left\langle\mathcal{A}_{\alpha}(v), \eta\right\rangle\right\rangle=0 \quad \forall \eta \in V_{p^{\prime}}
$$

insert the trial function $\eta=v_{\tau}$, where $v_{\tau}$ is defined according to (3.1). After passing to the limit $\tau \rightarrow 0$, we obtain

$$
\sigma \int_{\Gamma} \alpha|v| d S-\sigma \theta \int_{\Gamma} \operatorname{sign}(v) H(\alpha v) d S \leq 0 .
$$

If $\theta=0$, then $\alpha|v|=0$ on $\Gamma$; if $\theta=1$, by virtue of $H_{2}$ and Hölder inequality, we have

$$
\int_{\Gamma} \alpha|v| d S \leq q\|\alpha v\|_{L_{1}(\Gamma)}
$$

what is sufficient for $\alpha v=0$ on $\Gamma$. From here, our integral equality and invertibility of $\mathcal{A}_{0}$ now follows immediately that $v=0$.

If $p \leq 2$, then due the embedding theorems

$$
\alpha v \in L_{8 / 5}(\Gamma) \subset\left(V_{2}\right)^{*},
$$

and from our integral equality and invertibility of $\mathcal{A}_{0}$ it follows that $v \in V_{2}$. Now we can insert in our integral equality the function $v_{\tau}$ as the trial function $\eta$ and, analogously as above, we get $v=0$.

Theorem 1. Let the hypotheses $H_{0}-H_{3}$ hold. Then there exists a constant $c_{0}$, which depend only on $\mu$, such that for all operators $\mathcal{A}_{\alpha} \in \mathbf{A}(\mu)$ and all $p \in[4 / 3,4]$

$$
\left\|\mathcal{A}_{\alpha}^{-1}\right\| \leq c_{0} \text { in the norm of } \mathcal{L}\left(\left(V_{p^{\prime}}\right)^{*} \longrightarrow V_{p}\right)
$$

Proof. Due the interpolation theorem of Riesz, see, for instance, Dunford and Schwartz [5, Proposition 39, p. 579], it is sufficient to show the inequality (4.3) for $p=4 / 3$ and $p=4$.

Let us consider the case $p=4 / 3$ and let us suppose that (4.3) does not hold. That means there exist $d>0$ and sequences

$$
\left\{v_{k}\right\} \subset V_{4 / 3}, \quad\left\{\alpha_{k}\right\} \subset L_{\infty}(\Gamma) \quad \text { with } 0 \leq \alpha(x) \leq \mu \forall x \in \Gamma,
$$


such that

$$
\begin{gathered}
\sup _{\|\eta\|_{V_{4}} \leq 1}\left\langle\left\langle\mathcal{A}_{\alpha_{k}}\left(v_{k}\right), \eta\right\rangle\right\rangle=\frac{1}{k}, \\
\left\|v_{k}\right\|_{V_{4 / 3}}=1, \quad k=1,2, \ldots
\end{gathered}
$$

Without loss of generality, we can assume that due the embedding theorems the sequence $\left\{v_{k}\right\}$ converges strongly in $L_{7 / 5}(\Gamma)$ to some $v_{0}$ and we can assume also that the sequences $\left\{v_{k}\right\},\left\{\alpha_{k}\right\},\left\{\alpha_{k} v_{k}\right\}$ converges weakly to $v_{0}, \alpha_{0}, \alpha_{0} v_{0}$ in $V_{4 / 3}, L_{8}(\Gamma)$ and $L_{7 / 5}(\Gamma)$ respectively.

After passage to the limit $k \rightarrow \infty$ in (4.4) we have

$$
\left\langle\left\langle\mathcal{A}_{\alpha_{0}}\left(v_{0}\right), \eta\right\rangle\right\rangle=0 \quad \forall \eta \in V_{4}
$$

If $v_{0} \neq 0$, then we have a contradiction with Lemma 1 . If $v_{0}=0$, then by virtue of the convergence properties the terms in (4.4), which contain $\alpha_{k} v_{k}$ in (4.4) as elements of $\left(V_{4}\right)^{*}$ converge to zero strongly as $k \rightarrow \infty$ what gives

$$
\begin{gathered}
\sup _{\|\eta\|_{V_{4}} \leq 1}\left\langle\left\langle\mathcal{A}_{0} v_{k}, \eta\right\rangle\right\rangle \rightarrow 0 \text { as } k \rightarrow \infty, \\
\left\|v_{k}\right\|_{V_{4 / 3}}=1, \quad k=1,2, \ldots
\end{gathered}
$$

But that contradicts with Lemma 1.

\section{Further Properties of the Modified Operator}

The variational equality (3.3) defines the operator $F: V_{2} \rightarrow V_{2}^{*}$,

$$
\begin{aligned}
\langle\langle F, \eta\rangle\rangle:= & \int_{\Omega}\left[a\left\langle\nabla\left(u+u_{*}\right), \eta\right\rangle+b\left(u+u_{*}\right)_{x_{3}} \eta\right] d x \\
& +\sigma \int_{\Gamma}\left[(I-H)\left(\psi\left(u+u_{*}\right)\right)\right] \eta d S-\langle\langle f, \eta\rangle\rangle \quad \forall \eta \in V_{2} .
\end{aligned}
$$

By virtue of $H_{1}-H_{4}$ the operator $F$ is Frechet differentiable, for every $u \in V_{2}$ the Frechet derivative $F^{\prime}(u)$ belongs to $\mathbf{A}\left(\mu_{2}\right)$, and the mapping $u \rightarrow F^{\prime}(u)$ is uniformly Lipschitz continuous, i.e., there exists a constant $L$, which depends only on $\Omega, \Gamma, \sigma, \mu_{2}$ such that

$$
\left\|F^{\prime}\left(u_{1}\right)-F^{\prime}\left(u_{2}\right)\right\|_{\mathcal{L}\left(V_{2} \rightarrow V_{2}^{*}\right)} \leq L\left\|u_{1}-u_{2}\right\| \quad \forall u_{1}, u_{2} \in V_{2}
$$

More than that, Theorem 1 implies that there exists a constant $c_{0}$, which depends only on terms in $H_{1}-H_{3}$ and the constant $\mu_{1}$ from $H_{4}$, such that operators $F^{\prime}(u)$ are invertible and

$$
\left\|F^{\prime-1}(u)\right\| \leq c_{0} \quad \forall u \in V_{2} .
$$

Without loosing generality, we can assume that these estimates hold for operators $F$ with arbitrary operators $H$, which satisfy $H_{1}-H_{2}$, including the case $H \equiv 0$. 
Obtained properties allow to apply various damped Newton methods for solving (3.3).

For convenience of the reader and to illustrate how the constants $c_{0}$ and $L$ appear in the algorithm, which we propose, we present below the standard proof for the convergence properties for the damped Newton method. The reasoning, which we apply, in essence is the same as in R.E. Bank and D.J. Rose [2, Section 1], see also C.T. Kelley [7, Section 8].

Lemma 2. Let $X, Y$ be Banach spaces and let $\mathcal{F}: X \rightarrow Y$ be such that

(i) $\mathcal{F}$ is continuous and has the continuous Frechet derivative $\mathcal{F}^{\prime}(\cdot)$;

(ii) the operator $\mathcal{F}^{\prime}(\cdot)$ is invertible and there exists a constant $c_{0}$ such that

$$
\left\|\left(\mathcal{F}^{\prime}(x)\right)^{-1}\right\|_{\mathcal{L}(Y \rightarrow X)} \leq c_{0} \quad \forall x \in X
$$

(iii) there exist a constant $L$ such that

$$
\left\|\mathcal{F}^{\prime}\left(x_{1}\right)-\mathcal{F}^{\prime}\left(x_{2}\right)\right\|_{\mathcal{L}(X \rightarrow Y)} \leq L\left\|x_{1}-x_{2}\right\| \quad \forall x_{1}, x_{2} \in X .
$$

Then the iterative process

$$
\begin{gathered}
\mathcal{F}^{\prime}\left(x_{k}\right)\left[x_{k+1}-x_{k}\right]=-\lambda_{k} \mathcal{F}\left(x_{k}\right), \quad k=1,2, \ldots, \\
\lambda_{k}:=\min \left\{1 ; \frac{1}{2 L c_{0}^{2}\left\|\mathcal{F}\left(x_{k}\right)\right\|}\right\},
\end{gathered}
$$

for every fixed $x_{1}$ converges to the solution $x_{0}$ of the equation

$$
\mathcal{F}(x)=0
$$

with the rate of a geometric progression with the quotient

$$
\tilde{q} \leq \max \left\{\frac{1}{2} ; 1-\frac{1}{4 L c_{0}^{2}\left\|\mathcal{F}\left(x_{1}\right)\right\|}\right\} .
$$

Proof. From (5.1) and Taylor's formula we have

$$
\begin{aligned}
& \mathcal{F}\left(x_{k+1}\right)=\mathcal{F}\left(x_{k}\right)-\lambda_{k} \mathcal{F}\left(x_{k}\right) \\
& \quad+\int_{0}^{1}\left[\mathcal{F}^{\prime}\left(x_{k}+t\left(x_{k+1}-x_{k}\right)\right)-\mathcal{F}^{\prime}\left(x_{k}\right)\right]\left(x_{k+1}-x_{k}\right) d t, \\
& \left\|x_{k+1}-x_{k}\right\| \leq \lambda_{k} c_{0}\left\|\mathcal{F}\left(x_{k}\right)\right\|,
\end{aligned}
$$

what gives

$$
\left\|\mathcal{F}\left(x_{k+1}\right)\right\| \leq\left(1-\lambda_{k}\right)\left\|\mathcal{F}\left(x_{k}\right)\right\|+L c_{0}^{2} \lambda_{k}^{2}\left\|\mathcal{F}\left(x_{k}\right)\right\|^{2} .
$$

From this inequality and the choice of $\lambda_{k}$ immediately follows that

$$
\left\|\mathcal{F}\left(x_{k+1}\right)\right\| \leq\left\|\mathcal{F}\left(x_{k}\right)\right\| \begin{cases}1 / 2, & \text { if } L c_{0}^{2}\left\|\mathcal{F}\left(x_{k}\right)\right\| \leq 1 / 2, \\ 1-1 /\left(4 L c_{0}^{2}\left\|\mathcal{F}\left(x_{k}\right)\right\|\right), & \text { otherwise }\end{cases}
$$

From here, boundedness from below of $\lambda_{k},(5.1)$ and (ii) the statement of Lemma follows. 
Remark 1. Obviously, if the value of $\left\|\mathcal{F}\left(x_{1}\right)\right\|$ is sufficiently small, then (5.1) coincides with the Newton iteration process.

Remark 2. Hypothesis $H_{4}$ and Theorem 1 ensure that the variational equality (3.3) define an operator $F: V_{2} \rightarrow\left(V_{2}\right)^{*}$, for which the assumptions of Lemma 2 hold. Therefore, the equation (3.3) for every right hand side in $\left(V_{2}\right)^{*}$ has a solution $v \in V_{2}$. More than that, from $H_{4}$ it follows that for every chosen $v, u \in V_{2}$

$$
\int_{0}^{1} F^{\prime}(u+t v) d t \in \mathbf{A}(\mu)
$$

with $\mu=\mu_{1}$ and $\theta=1$. That is sufficient for the uniqueness of solutions of $(3.3)$.

\section{Iterative Process}

We propose the following two level iterative process. Consider the operator $F: V_{2} \rightarrow\left(V_{2}\right)^{*}$ corresponding to our modified variational equality (3.3),

$$
\begin{aligned}
\langle\langle F(u), \eta\rangle\rangle:= & \int_{\Omega}\left[a\left\langle\nabla\left(u+u_{*}\right), \nabla \eta\right\rangle+b\left(u+u_{*}\right)_{x_{3}} \eta\right] d x \\
& +\sigma \int_{\Gamma}\left[\psi\left(u+u_{*}\right)-H\left(\psi\left(u+u_{*}\right)\right)\right] \eta d S-\langle\langle f, \eta\rangle\rangle \quad \forall \eta \in V_{2},
\end{aligned}
$$

where $f \in\left(V_{2}\right)^{*}$ is fixed and the function $\psi$ satisfies $H_{4}$.

The first level of iterations consists of

$$
\begin{aligned}
\int_{\Omega} & {\left[a\left\langle\left(\nabla v_{k+1}-\nabla v_{k}, \nabla \eta\right\rangle+b\left(v_{k+1 x_{3}}-v_{k x_{3}}\right) \eta\right] d x\right.} \\
& +\sigma \int_{\Gamma} \psi^{\prime}\left(v_{k}+u_{*}\right)\left(v_{k+1}-v_{k}\right) \eta d S \\
= & \lambda_{k}\left\{\int_{\Omega}\left[a\left\langle\nabla v_{k}, \nabla \eta\right\rangle+b v_{k x_{3}} \eta\right] d x+\sigma \int_{\Gamma} \psi\left(v_{k}+u_{*}\right) \eta d S-\left\langle\left\langle f_{m}, \eta\right\rangle\right\rangle\right\} \\
\forall & \forall \in V_{2}, k=1,2, \ldots,
\end{aligned}
$$

with some $f_{m} \in\left(V_{2}\right)^{*}$, where the parameter $\lambda_{k}$ is chosen according to Lemma 2 for the operator $F_{0}: V_{2} \rightarrow V_{2}^{*}$,

$$
\left\langle\left\langle F_{0}(v), \eta\right\rangle\right\rangle:=\int_{\Omega}\left[a\left\langle\nabla\left(v+u_{*}\right), \nabla \eta\right\rangle+b\left(v+u_{*}\right)_{x_{3}} \eta\right] d x+\sigma \int_{\Gamma} \psi\left(v+u_{*}\right) \eta d S .
$$

The second level of iterations consists of

$$
\begin{gathered}
\int_{\Omega}\left[a\left\langle\nabla\left(u_{m+1}+u_{*}\right), \nabla \eta\right\rangle+b\left(u_{m+1}+u_{*}\right)_{x_{3}} \eta\right] d x+\sigma \int_{\Gamma} \psi\left(u_{m+1}+u_{*}\right) \eta d S \\
=\sigma \int_{\Gamma} H\left(\psi\left(u_{m}+u_{*}\right)\right) \eta d S-\langle\langle f, \eta\rangle\rangle \quad \forall \eta \in V_{2}, m=1,2, \ldots
\end{gathered}
$$

The iteration process $(6.2)$ gives the passage from $u_{m}$ to $u_{m+1}$ in the iteration process (6.3). 
Here we treat the solution of the equation $\mathcal{A}_{\alpha}(v)=l, l \in V_{2}^{*}$ with $\theta=0$ as a basic procedure. It is clear, that we can not avoid something alike as the realization of the projection operator $\Pi: L_{2}\left(\Omega ; \mathbb{R}^{3}\right) \rightarrow\left\{\bar{f} \in L_{2}\left(\Omega ; \mathbb{R}^{3}\right) \mid\right.$ $\left.\bar{f}=\nabla u, u \in V_{2}\right\}$ and the realization of $\mathcal{A}_{\alpha}^{-1}$ with $\theta=0$ is very similar to the realization of $\Pi$.

It remains to show that the iteration process (6.3) converges. Let us denote

$$
\delta_{m+1}:=u_{m+1}-u_{m}, \omega_{m+1}:=\psi\left(u_{m+1}+u_{*}\right)-\psi\left(u_{m}+u_{*}\right), \quad m=1,2, \ldots
$$

Then (6.3) gives

$$
\int_{\Omega}\left[a\left\langle\nabla \delta_{m+1}, \nabla \eta\right\rangle+b \delta_{m+1 x_{3}} \eta\right] d x+\sigma \int_{\Gamma} \omega_{m+1} \eta d S=\sigma \int_{\Gamma} H\left(\omega_{m}\right) \eta d S .
$$

By inserting in (6.4) as trial functions $\eta=\left(\delta_{m+1}\right)_{\tau}$, defined according to (3.1), and passing to the limit $\tau \rightarrow 0$ we have

$$
\sigma \int_{\Gamma}\left|\omega_{m+1}\right| d S \leq \sigma \int_{\Gamma} \operatorname{sign}\left(\delta_{m+1}\right) H\left(\omega_{m}\right) d S
$$

From here and $H_{2}$ it follows immediately that

$$
\left\|\omega_{m+1}\right\|_{L_{1}(\Gamma)} \leq q\left\|\omega_{m}\right\|_{L_{1}(\Gamma} \leq q^{m-1}\left\|\omega_{1}\right\|_{L_{1}(\Gamma)}, \quad m=1,2, \ldots
$$

Because $L_{1}(\Gamma) \subset V_{4 / 3}^{*}$ we can apply Theorem 1 to (6.3) (treating $\omega_{m+1}$ and $\omega_{m}$ as known parameters), what gives that

$$
\left\|\delta_{m+1}\right\|_{V_{4 / 3}} \leq c_{1} q^{m-1}, \quad m=1,2, \ldots,
$$

where the constant $c_{1}$ does not depend on $m$.

In turn, from $H_{4}$ it follows that $\left|\omega_{m}\right| \leq \mu_{1}\left|\delta_{m}\right|$, what together with the embedding theorems ensure that elements $\omega_{m+1}$ and $H\left(\omega_{m}\right)$ belong to $V_{2}^{*}$ and their norms in this space are majored by $c_{2} q^{m-1}$ and $c_{2} q^{m-2}$ respectively, where the constant $c_{2}$ does not depend on $m$.

Now, from Theorem 1 it follows that there is

$$
\left\|\delta_{m+1}\right\|_{V_{2}}=\left\|u_{m+1}-u_{m}\right\|_{V_{2}} \leq c_{3} q^{m-2}, \quad m=2,3, \ldots,
$$

what is sufficient for the convergence of the sequence $\left\{u_{m}\right\}$ in $V_{2}$ to the solution of the equation (3.3).

\section{Numerical Tests}

To illustrate performance of the iterative procedure described in the previous section we carried out some numerical tests for a real life problem taken from [4], where treatment of glass fabric sheets at high temperature is modeled.

Problem setup we took almost the same as in case of the original problem. By $\Omega:=(-0.55,0.55) \times(-0.0001,0.0001) \times(0,3)$ we denote thin glass fabric sheet, which is treated at high temperature in a special furnace. Geometry of the furnace we took very simple:

$$
\Omega_{f}:=((-0.715,0.715) \times(-0.15,0.2) \times(0,3)) \backslash \bar{\Omega} .
$$


Table 1. Inner and outer iterations.

\begin{tabular}{|c|c|c|c|c|c|c|}
\hline \multirow[t]{2}{*}{ Residual } & \multicolumn{2}{|c|}{$\begin{array}{c}m=1 \\
68480.47\end{array}$} & \multicolumn{2}{|c|}{$\begin{array}{c}m=2 \\
13896.73\end{array}$} & \multicolumn{2}{|c|}{$\begin{array}{c}m=3 \\
2928.82\end{array}$} \\
\hline & Residual & $\lambda_{k}$ & Residual & $\lambda_{k}$ & Residual & $\lambda_{k}$ \\
\hline$k=1$ & 68480.47 & 0.29 & 13896.73 & 1 & 2928.82 & 1 \\
\hline$k=2$ & 55044.67 & 0.36 & 1075.27 & 1 & 37.38 & 1 \\
\hline$k=3$ & 36660.21 & 0.54 & 5.43 & 1 & 0.007 & 1 \\
\hline$k=4$ & 18467.04 & 1 & 0.0005 & 1 & 0.0005 & 1 \\
\hline$k=5$ & 3249.37 & 1 & - & - & - & - \\
\hline$k=6$ & 274.20 & 1 & - & - & - & - \\
\hline$k=7$ & 2.63 & 1 & - & - & - & - \\
\hline$k=8$ & 0.0005 & 1 & - & - & - & - \\
\hline
\end{tabular}

By $\Gamma_{h}:=\left(\partial \Omega_{f} \backslash \partial \Omega\right) \cap\left\{0.1 \leq x_{3} \leq 1.3\right\}$, we denote a heater of the furnace, where about $1100 \mathrm{~K}$ high temperature is applied. Let us also define:

$$
\Gamma_{0}:=\bar{\Omega} \cap\left\{x_{3}=0\right\}, \quad \Gamma_{1}:=\bar{\Omega} \cap\left\{x_{3}=3\right\}, \quad \Gamma:=\partial \Omega \backslash\left(\Gamma_{0} \cup \Gamma_{1}\right)
$$

and $a:=1.38, b:=250767.66, \sigma:=5.67 \times 10^{-8}, \epsilon:=0.91$.

To model temperature of the fabric sheet various aspects of heat transfer must be encountered in the model. Heat transfer by conduction and convection take place in $\Omega_{f}$ due to pulling of the sheet with constant speed through the furnace. At the same time, considerable radiative heat flux exists on the fabricair interface $\Gamma$ and the heater-air interface $\Gamma_{h}$ due transparency of air for heat radiation and high working temperature of the heater. Nevertheless, it is easy to see, that previously defined problem easily fits under the model problem (1.1).

It is also clear that for described test problem hypotheses $H_{0}-H_{3}$ are satisfied. Indeed, $\Omega$ is a rectangular parallelepiped, therefore $H_{0}$ holds. Properties $H_{1}, H_{2}$ can be proven by techniques proposed by M. Laitinen and T. Tiihonen in [9], where it is shown that, if $\Gamma$ is not a closed surface, then $\|H\|_{\mathcal{L}\left(L_{p}(\Gamma) \rightarrow L_{p}(\Gamma)\right)}=q<1$ for every $1 \leq p \leq \infty$. Obviously, $H_{3}$ is also satisfied.

For discretization of the test problem described above we used finite volume approach (see [11]). Our example has only illustrative character, therefore the accuracy of approximations was not the issue here and we used only $36 \times 5 \times 108$ cell centered grid for discretization of the problem.

In Table 1 we summarized convergence results for few starting outer iterations. As it is easy to see from these results, if residual of inner iterations exceeds certain level, then calculated $\lambda_{k}$ is less that 1 and overall convergence rate is relatively slow (as for the case $m=1$ ). Nevertheless, after residual drops to certain level, $\lambda_{k}$ becomes equal to 1 and following inner iterations become pure Newton iterations making further convergence very fast.

\section{References}

[1] R.A. Adams. Sobolev Spaces. Academic Press, New York, 1975.

[2] R.E. Bank and D.J. Rose. Global approximate Newton methods. Numer. Math., 37:279-295, 1981. http://dx.doi.org/10.1007/BF01398257. 
[3] K. Birgelis. Optimal control in models with conductive-radiative heat transfer. Math. Model. Anal., 8(1):1-12, 2003. http://dx.doi.org/10.1080/13926292.2003.9637205.

[4] A. Buikis and A. D. Fitt. A mathematical model for the heat treatment of glass fabric sheets. IMA Journal of Mathematics Applied in Business and Industry, 10(1):55-86, 1999. http://dx.doi.org/10.1093/imaman/10.1.55.

[5] N. Dunford and J. T. Schwartz. Linear Operators I, General Theory. Interscience Publishers, New York, 1958.

[6] A. Fröhlich. The Helmholtz decomposition of weighted $L_{q}$-spaces for Mackenhoupt weights. Ann. Univ. Ferrara Sez. VII Sci. Mat., 46:11-19, 2000.

[7] C.T. Kelley. Iterative Methods for Linear and Nonlinear Operators. SIAM, Phyladelphia, 1995.

[8] D. Krause and H. Loch (Eds.). Mathematical Simulation in Glass Technology. Springer-Verlag, Berlin, 2002.

[9] M. Laitinen and T. Tiihonen. Conductive-radiative heat transfer in grey materials. Quart. Appl. Math., 59(4):737-768, 2001.

[10] C. Mayer, P. Philip and F. Tröltzsch. Optimal control of a semilinear PDE with nonlocal radiation interface conditions. SIAM J. Control Optim., 45:699-721, 2006. http://dx.doi.org/10.1137/040617753.

[11] W.J. Minkowycz, E.M. Sparrow and J.Y. Murthy. Handbook of Numerical Heat Transfer. Wiley, Hoboken, 2006. 